\title{
Deviations from Matthiessen's Rule for $\mathrm{SrRuO}_{3}$ and $\mathrm{CaRuO}_{3}$
}

\author{
L. Klein, Y. Kats, and N. Wiser \\ Department of Physics, Bar-Ilan University, Ramat-Gan 52900, Israel \\ M. Konczykowski \\ Laboratoire des Solides Irradies, Ecole Polytechnique, 91128 Palaiseau Cedex, France \\ J. W. Reiner, T. H. Geballe, M. R. Beasley, and A. Kapitulnik \\ Edward L. Ginzton Laboratories, Stanford University, Stanford, California 94305
}

(November 15, 2018)

\begin{abstract}
We have measured the change in the resistivity of thin films of $\mathrm{SrRuO}_{3}$ and $\mathrm{CaRuO}_{3}$ upon introducing point defects by electron irradiation at low temperatures, and we find significant deviations from Matthiessen's rule. For a fixed irradiation dose, the induced change in resistivity decreases with increasing temperature. Moreover, for a fixed temperature, the increase in resistivity with irradiation is found to be sublinear. We suggest that the observed behavior is due to the marked anisotropic scattering of the electrons together with their relatively short mean free path (both characteristic of many metallic oxides including cuprates) which amplify effects related to the Pippard ineffectiveness condition.
\end{abstract}

Typeset using REVTEX 
Deviations from Matthiessen's rule (DMR) in a metal provide unique microscopic insight into the electron scattering processes. Here, we have used electron irradiation to study the DMR in the ruthenium-based perovskites $\mathrm{SrRuO}_{3}$ and $\mathrm{CaRuO}_{3}$. These ruthenates have been studied extensively in recent years, 10 with various experimental results indicating that these are strongly correlated systems, similar in many ways to the high- $T_{c}$ cuprates. Therefore, there is special interest in exploring the DMR in these systems.

Usually, the DMR have been studied by comparing the electrical resistivities of samples containing different concentrations of impurities. 6 However, this method may induce changes in the resistivity that are not related to the concentration of point defects, thus limiting the possibility of a rigorous and sensitive study of the DMR. On the other hand, it has been pointed out 8 that examining the resistivity change of a sample whose residual resistivity is moderately increased by electron irradiation would allow a detailed study of the DMR which could be compared with theoretical predictions.

$\mathrm{SrRuO}_{3}$ and $\mathrm{CaRuO}_{3}$ are pseudo-cubic perovskites having similar electronic structure and comparable resistivities. Nevertheless, the slight difference in their lattice parameters leads to important differences in their magnetic behavior. $\mathrm{SrRuO}_{3}$ is an itinerant ferromagnet with $T_{c} \sim 150 \mathrm{~K}$ (in films), whereas $\mathrm{CaRuO}_{3}$ does not exhibit long-range order down to at least $1.8 \mathrm{~K}$. Since $\mathrm{SrRuO}_{3}$ and $\mathrm{CaRuO}_{3}$ are very similar except for magnetic ordering, studying both systems allows us to determine whether the observed DMR are due to the magnetic contribution.

Our measurements show significant DMR for both $\mathrm{SrRuO}_{3}$ and $\mathrm{CaRuO}_{3}$. The experimental quantity of interest is the change in resistivity $(\rho)$ upon electron irradiation, $\Delta \rho_{\text {irr }}(D, T)=\rho(D, T)-\rho(0, T)$, where $D$ is the irradiation dosage and $T$ is the temperature. We measured $\Delta \rho_{i r r}(D, T)$ as a function of $D$ for fixed $T$ and as a function of $T$ for fixed $D$. Our principal results are the following: (i) for fixed dosage, $\Delta \rho_{\text {irr }}$ decreases with increasing temperature (see Fig. 1), and (ii) for fixed temperature, $\Delta \rho_{\text {irr }}$ increases sublinearly as a function of dosage (see Fig. 2). We explain these results in terms of anisotropic electron scattering (due to the anisotropic Fermi surfacel 1 ) together with the Pippard "ineffective- 
ness condition" 911 for low- $q$ scattering due to a short mean free path. Since anisotropic scattering and a short mean free path are also characteristic of other metallic perovskites (including the cuprates), DMR may be expected to occur in these systems as well.

The samples used for electron irradiation were $\mathrm{SrRuO}_{3}$ films grown on $\mathrm{SrTiO}_{3}$ and a $\mathrm{CaRuO}_{3}$ film grown on $\mathrm{NdGaO}_{3}$ (different substrates where chosen to minimize the lattice mismatch). The samples were irradiated by a beam of $2.5 \mathrm{MeV}$ electrons using the VINKAC set-up at Ecole Polytechnique, composed of a Van de Graff accelerator and an irradiation chamber connected to a closed-cycle hydrogen liquefier. Electrons of such energy are known 12 to create point defects in compounds similar to the perovskites used in our experiment. During irradiation, the samples were immersed in liquid hydrogen (20 K) to prevent diffusion and clustering of the generated point defects. In the first warm-up, we observe a small decrease in $\Delta \rho_{i r r}$ which indicates some defect migration when the temperature is increased. By analogy with other electron-irradiated systems (e.g., $\mathrm{YBa}_{2} \mathrm{Cu}_{3} \mathrm{O}_{7}$ ) whose defects were extensively studied with a tunneling-electron microscope, this decrease is due to partial annealing or the formation of small clusters which are equivalent to point defects. The defects remained stable in subsequent temperature cycles.

Prior to irradiation, the films were patterned to allow resistivity measurements on pairs of neighboring segments. During irradiation, one segment of each pair was covered with lead to protect it from being irradiated. Therefore, we could determine the effect of irradiation by comparing the resistivities of neighboring segments, with the lead-covered segment serving as reference. The fact that the comparison is made between segments that are identical except for the irradiation, and the fact that the resistivity of the two segments was measured simultaneously (thus avoiding errors due to small variations in the sample temperature at the same nominal temperature settings during different measurement runs) are crucial for reliable and sensitive measurements of the small changes in resistivity due to irradiation.

The main results of this paper are presented in Figs. 1 and 2. Figure 1 shows the temperature dependence of $\Delta \rho_{\text {irr }}$ at a fixed dosage for $\mathrm{SrRuO}_{3}$ and $\mathrm{CaRuO}_{3}$. At low temperatures, $\Delta \rho_{\text {irr }} \sim 3 \mu \Omega \mathrm{cm}$ for both samples, but as the temperature increases, $\Delta \rho_{\text {irr }}$ decreases sig- 
nificantly. The similarity in the behavior of $\Delta \rho_{\text {irr }}$ for $\mathrm{SrRuO}_{3}$ and $\mathrm{CaRuO}_{3}$ indicates that the observed DMR is not caused by magnetic ordering. However, the pronounced "step" in $\Delta \rho_{\text {irr }}$ near the Curie temperature of $\mathrm{SrRuO}_{3}$ suggests that the magnetic ordering does affect $\Delta \rho_{i r r}$.

Figure 2 shows $\rho$ as a function of dosage for $\mathrm{SrRuO}_{3}$ at $20 \mathrm{~K}$. Whenever the density of the added point defects is proportional to the dosage, 13 one expects a linear increase in $\Delta \rho_{i r r}$. However, we find a sublinear dependence; the inset clearly shows a monotonic decrease in $d \rho / d D$

We suggest that the key to understanding these results is twofold: (i) the scattering is very anisotropic for the ruthenates; therefore, even small-angle scattering can produce large changes in the velocity of the electron, and (ii) the anisotropic scattering amplifies the Pippard "ineffectiveness condition" 9 which states that the low- $q$ components of scattering potentials are ineffective in scattering when $1 / q$ is larger than the electron mean free path.

The physics behind the ineffectiveness condition is straightforward. A finite mean free path $\lambda$ implies that the $k$-vector of the electron has an uncertainty of magnitude $\Delta k \sim 1 / \lambda$. Therefore, if the electron $k$-vector changes upon scattering by less than $\Delta k$, then the initial and final $k$-vectors are the same within their uncertainty. This is equivalent to the electron not having been scattered at all.

The change in the electron $k$-vector upon scattering is given by the $q$-vector of the scattering potential, $V_{q}$. The ineffectiveness condition implies that the mean free path $\lambda$ sets a lower bound $q_{\text {min }}=2 \pi / \lambda$, so that $V_{q}$ with $q<q_{\text {min }}$ is excluded as a scattering source.

The ineffectiveness condition explains the behavior of $\Delta \rho_{i r r}(D, T)$, both as a function of temperature (Fig. 1) and as a function of irradiation dosage (Fig. 2).

Two different contributions determine the temperature-dependent decrease of $\Delta \rho_{\text {irr }}$. First, because $q_{\min }$ increases as a function of temperature, the resistivity due to the added defects becomes smaller. Second, the irradiation-induced shortening of the mean free path makes more small-q phonons and magnons "ineffective", thus yielding a negative contribution to $\Delta \rho_{i r r}$. 
Similarly, the sublinear increase of $\Delta \rho_{i r r}$ with dosage is explained by the decrease of the contribution per defect to $\rho$ due to the progressive increase in $q_{\min }$.

Why are similar DMR effects not observed in all metals? For metals having a nearlyspherical Fermi surface, the small- $q$ components of the scattering potential contribute very little to the resistivity due to the $(1-\cos \theta)$ "transport factor" ( $\theta$ is the scattering angle). The approximate expression $(1-\cos \theta) \propto q^{2}$ for the transport factor reflects the fact that in simple metals, small scattering angles imply small changes in electron velocity (hence, current). On the other hand, for a material with an anisotropic Fermi surface, even smallangle scattering can lead to a large change in the electron velocity. As a result, the transport factor is of order unity.14

The effect of a non-spherical Fermi surface has been calculated, for instance, for aluminium. 1 . Using the correct transport factor, which turns out to be of order unity, accounts for the observed low-temperature $T^{3}$ dependence for $\rho(T)$, rather than the usual $T^{5}$ dependence.

For the materials studied here, $d$-electrons are mainly responsible for the current and the electron scattering is very anisotropic. Therefore, similar to aluminium, the transport factor is expected to be of order unity over much of the non-spherical Fermi surface. Moreover, these materials are characterized by a short mean free path which implies large $q_{\min }$.

We now consider the magnitude of $\Delta \rho_{i r r}$. The experimental results for $\mathrm{SrRuO}_{3}$ and $\mathrm{CaRuO}_{3}$ are similar, and we shall concentrate on the data for $\mathrm{SrRuO}_{3}$. According to Fig. 1a, as the temperature increases to $100 \mathrm{~K}$, the value of $\Delta \rho_{i r r}$ decreases from $3.0 \mu \Omega \mathrm{cm}$ (its low-temperature value) to $1.4 \mu \Omega \mathrm{cm}$. This $1.6 \mu \Omega \mathrm{cm}$ decrease in $\Delta \rho_{\text {irr }}$ is the number we wish to calculate.

An explicit resistivity calculation requires the scattering matrix elements and the complete phonon and magnon spectrum, and performing integrals over the anisotropic Fermi surface. None of these ingredients are known with sufficient precision. Therefore, we shall adopt the more modest goal of carrying out a simplified resistivity calculation to see whether we obtain reasonable agreement with experiment. To that end, we use the Bloch-Gruneisen 
approximation for both the electron-phonon and the electron-magnon resistivity. Although such a calculation will not yield quantitatively reliable numbers, we are here looking for a qualitative explanation of the behavior of $\Delta \rho_{i r r}$, rather than attempting a first-principles calculation.

Following the textbooks, 6 we write the Bloch-Gruneisen resistivity as follows:

$$
\rho=C \int_{q_{\min }(D, T)}^{Q} d q q|V(q)|^{2} F\left[\hbar \omega(q) / k_{B} T\right] ; \quad F(z)=\frac{z^{2}}{\left(e^{z}-1\right)\left(1-e^{-z}\right)}
$$

where $q_{\min }(D, T)$ depends on both the dosage $D$ and the temperature through its dependence on the resistivity, and the upper limit is the Debye wavenumber $Q$. At low $q$ (the region of interest here), $\omega(q) \propto q$ for phonons, whereas for magnons, $\omega(q) \propto q^{2}$. The Debye temperature and some other parameters are different, of course, for phonons and for magnons. For the form factor $V(q)$, which describes the scattering of an electron by a unit cell, we used the $q$-dependence which has been calculated 7 for the simple metals. We used the kinetic theory of metals 18 to relate $\rho$ to $\lambda$ for the calculation of $q_{\min }=2 \pi / \lambda$.

Equation (11) differs from the textbook expression in two respects. First, we have approximated the transport factor by unity. Second, the lower limit on the integral is $q_{\min }(D, T)$, rather than zero, which takes into account the ineffectiveness condition.

The resistivity of the defects is also given by Eq. (1), if one omits the function $F(z)$ and extends the range of integration to twice the Fermi momentum, which can be estimated from the calculated ${ }^{3}$ Fermi surface area.

Performing the calculation according to Eq. (11) yields a decrease in $\Delta \rho_{i r r}$ of $1.1 \mu \Omega \mathrm{cm}$ at $100 \mathrm{~K}$. Half of this value results from the decrease in the resistivity of the added defects from its zero-temperature value, and the other half results from the decrease in the phonon and magnon resistivities due to the irradiation-induced increase of $q_{\min }$. The calculated value of $1.1 \mu \Omega \mathrm{cm}$ is remarkably close to the experimental value of $1.6 \mu \Omega \mathrm{cm}$, in view of the crudity of the calculation, and should not be taken seriously as a quantitative result. However, it demonstrates that the ineffectiveness condition does indeed account for the magnitude of 
the observed decrease in $\Delta \rho_{i r r}$ with temperature.

At high temperatures our semi-classical treatment of $\rho_{i r r}$ is not applicable, since there are clear indications for the breakdown of Boltzmann transport theory in this regime. 1 回国 Therefore, a different theoretical approach is required.

A puzzling feature of the temperature dependence of $\Delta \rho_{i r r}$ that still needs to be explained is the sharp "step" in $\Delta \rho_{i r r}$ of $\mathrm{SrRuO}_{3}$ near $T_{c} \sim 153 \mathrm{~K}$ (see Fig. 1a). While one can attribute a decrease in $\Delta \rho_{i r r}$ near $T_{c}$ to the rapid increase of the magnetic resistivity, the sharp "step" is surprising because the magnetization approaches zero gradually, as the temperature approaches $T_{c}$ from below. Furthermore, when comparing the resistivity of the irradiated and unirradiated segments in magnetic field (see Fig. 3), additional unexpected behavior is observed near $T_{c}$. Attributing the "step" in $\Delta \rho_{i r r}$ to the decrease in the magnetization, the sharp "step" should be smeared out by the field. This was indeed observed, as shown in the figure, but we also found the unexpected result that below $T_{c}$, the application of the field reduces $\Delta \rho_{\text {irr }}$.

Both anomalies are resolved by assuming that the irradiation slightly decreases $T_{c}$ by about $\Delta T_{c} \sim 0.4 \mathrm{~K}$, which rescales the temperature dependence of the magnetic resistivity. Denoting the magnetic resistivity before and after irradiation by $\rho_{m}$ and $\rho_{m}^{\prime}$, respectively, we have near $T_{c}$ the relation $\rho_{m}^{\prime}(T, H) \simeq \rho_{m}\left(T+\Delta T_{c}, H\right)$. This change in the magnetic resistivity makes a temperature dependent contribution to $\Delta \rho_{i r r}$ that can be evaluated by determining $d \rho_{m} / d T .19$ Subtracting this contribution from $\Delta \rho_{i r r}$ yields the results shown in the upper inset of Fig. 3. These results indicate that below $T_{c}$, as expected, the field does not reduce $\Delta \rho_{i r r}$, and that the decrease of $\Delta \rho_{i r r}$ below $T_{c}$ is rather smooth.

The assumption that $T_{c}$ decreased by $\Delta T_{c} \sim 0.4 \mathrm{~K}$ due to irradiation is supported by the observed relation between $T_{c}$ and the residual resistivity in our unirradiated films. Furthermore, if we measure the magnetoresistance of the irradiated and unirradiated segments as a function of temperature, we see that the two curves coincide almost exactly if we shift one relative to the other by $\sim 0.4 \mathrm{~K}$ (see lower inset of Figure 3 ).

Turning now to Fig. 2, the inset presents the data for $d \rho / d D$, which gives a quantitative 
measure of the sublinearity of $\Delta \rho_{i r r}$. As the resistivity increases upon irradiation from 18.4 to $23.1 \mu \Omega \mathrm{cm}$, the value of $d \rho / d D$ is seen to decrease by $15 \%$. This is the number we wish to calculate.

Differentiating the resistivity integral with respect to $D$ for each contribution to the resistivity (phonons do not contribute at low temperatures) yields $3 \%$ for the decrease in $d \rho / d D$ upon irradiation. This value is much smaller than the experimental value of $15 \%$. However, the resistivity integral in (11) assumes isotropic electron scattering. If the marked anisotropy of the scattering over the Fermi surface is approximately taken into account, numerical studies indicate that the calculated value of $d \rho / d D$ increases by a factor of 2-3. (At the higher temperature of $100 \mathrm{~K}$, the effect is only marginal, because larger values of $q_{\text {min }}$ are involved.) Thus, a more realistic calculation of $d \rho / d D$ would yield about half the observed sublinearity of $\Delta \rho_{i r r}$.

In conclusion, we have clearly demonstrated that $\mathrm{SrRuO}_{3}$ and $\mathrm{CaRuO}_{3}$ exhibit significant DMR. We suggest that this behavior stems from the Pippard ineffectiveness condition, whose effect on the resistivity of these materials is amplified by their anisotropic scattering and short mean free path. We believe that these results will contribute to a better understanding of the transport properties of the compounds studied, as well as other similar systems.

We thank J. S. Dodge for useful discussions. This research was supported by The Israel Science Foundation founded by the Israel Academy of Sciences and Humanities and by Grant No. 97-00428/1 from the United States-Israel Binational Science Foundation (BSF), Jerusalem, Israel. 


\section{REFERENCES}

${ }^{1}$ P. B. Allen et al., Phys. Rev. B 53, 4393 (1996).

${ }^{2}$ I. I. Mazin and D. J. Singh, Phys. Rev. B 56, 2556 (1997)

${ }^{3}$ G. Santi and T. Jarlborg, J. Phys.: Condens. Matt. 9, 9563 (1997).

${ }^{4}$ L. Klein et al., Phys. Rev. Lett. 77, 2774 (1996); P. Kostic et al., Phys. Rev. Lett. 81, 2498 (1998); J.S. Dodge et al., Phys. Rev. Lett. (in press).

${ }^{5}$ L. Klein et al., Phys. Rev. B 60, 1448 (1999).

${ }^{6}$ G. Cao et al., Phys. Rev. B 56, 321 (1997). L. Klein et al., Phys. Rev. B 60, 1448 (1999).

${ }^{7}$ J. Bass, Adv. Phys. 21, 431 (1972).

${ }^{8}$ L. B. Ioffe and A. J. Millis, Phys. Rev. B 58, 11631 (1998).

${ }^{9}$ A. B. Pippard, Philos. Mag. 46, 1104 (1955); J. M. Ziman, Electrons and Phonons, (Clarendon Press, Oxford, 1960), p. 213.

${ }^{10}$ P. J. Cote and L. V. Meisels, Phys. Rev. Lett. 40, 1586 (1978); L. V. Meisels and P. J. Cote, Phys. Rev. B 30, 1743 (1984); J. Laakkonen and R. M. Nieminen, Phys. Rev. B 34, $567(1986)$.

${ }^{11}$ N. Wiser, J. Phys. F 18, 457 (1988); M. Kaveh and N. Wiser, Phys. Rev. B 36, 6339 (1987).

${ }^{12}$ H. Vichery et al., Physica C 159, 697 (1989).

${ }^{13}$ This is true as long as the density of defects is not too large and the probability of clustering of defects is small.

${ }^{14}$ M. Kaveh and N. Wiser, Phys. Rev. B 21, 2278 (1980).

${ }^{15}$ M. Kaveh and N. Wiser, Phys. Rev. B 21, 2291 (1980). 
16 J. M. Ziman, Electrons and Phonons (Clarendon Press, Oxford, 1960), p. 364.

${ }^{17}$ W. A. Harrison, Pseudopotentials in the Theory of Metals, (Benjamin, New York, 1966), p. 35 .

${ }^{18}$ N. W. Ashcroft and N. D. Mermin, Solid State Physics, (Saunders College Publishing, 1976), p. 52, Equation (2.91).

19 The behavior of $d \rho_{m} / d T$ near $T_{c}$ is determined by subtracting the derivative of the nonmagnetic components from the derivative of the total resistivity $(d \rho / d T)$. The derivative of the non-magnetic components is found by extrapolation from temperatures high enough above $T_{c}$ that $\rho_{m}$ is constant. 


\section{FIGURES}

FIG. 1. Resistivity change due to irradiation $\left(\Delta \rho_{\text {irr }}\right)$ for thin films of (a) $\mathrm{SrRuO}_{3}$ and (b) $\mathrm{CaRuO}_{3}$ as a function of temperature. The dosage for both samples is about $5 \times 10^{18}$ electrons $/ \mathrm{cm}^{2}$. The insets show the resistivity of the films before irradiation.

FIG. 2. In situ resistivity $(\rho)$ of a $\mathrm{SrRuO}_{3}$ film during irradiation as a function of dosage $(D)$. During irradiation, the sample was immersed in liquid hydrogen. The inset shows the derivative of the resistivity with respect to the dosage $(d \rho / d D)$ as a function of dosage.

FIG. 3. Resistivity change due to irradiation $\left(\Delta \rho_{i r r}\right)$ of a $\mathrm{SrRuO}_{3}$ film in zero field (open circles) and in a magnetic field of $1 \mathrm{~T}$ (full circles). The lower inset shows the magnetoresistance $(\mathrm{MR}=\rho(H)-\rho(0))$ of the unirradiated (full circles) and the irradiated segments (crosses). The upper inset shows $\Delta \rho_{i r r}^{*}$ which is $\Delta \rho_{i r r}$ with the change in $T_{c}$ (see text) taken into account. 


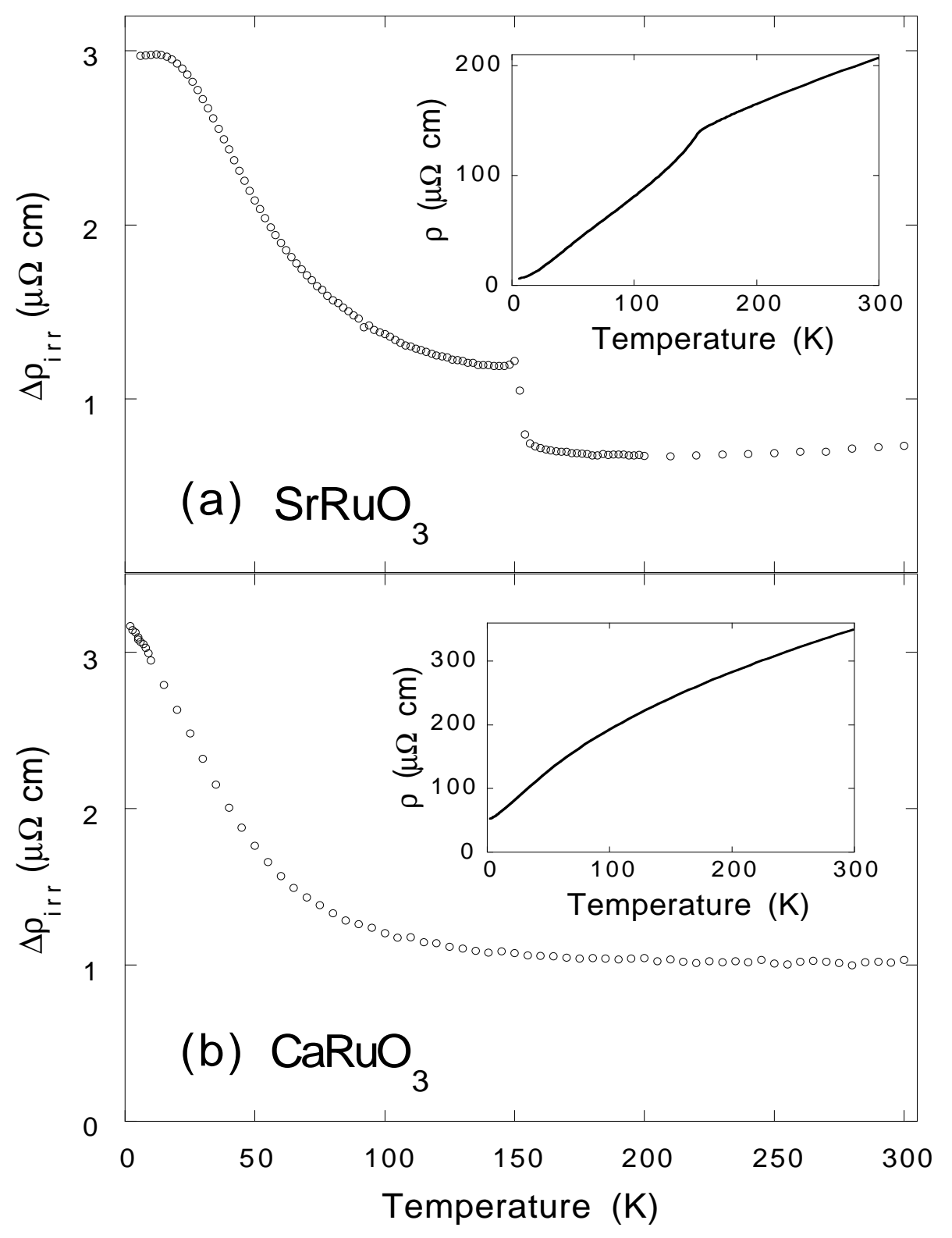

Figure 1, Klein et al 


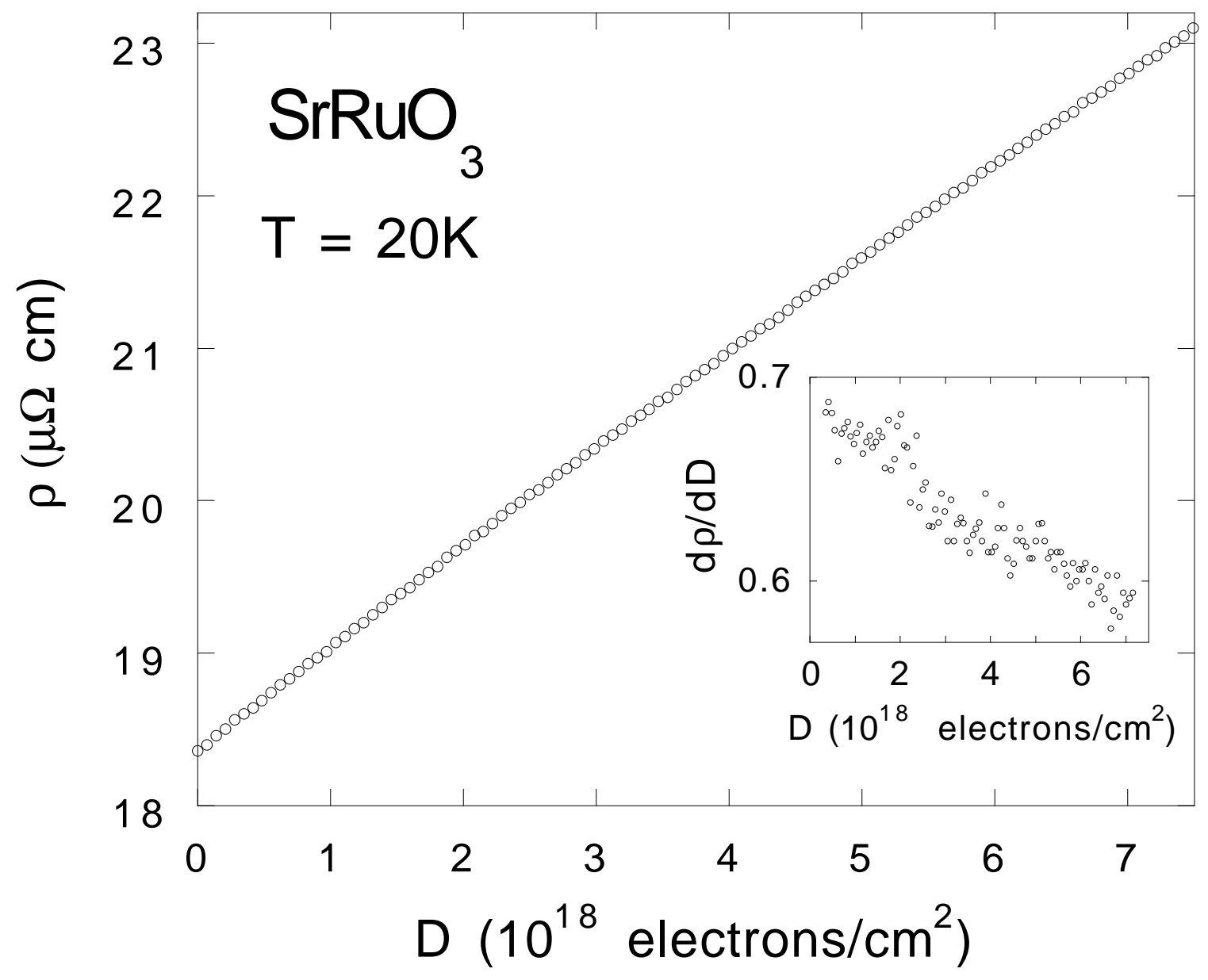

Figure 2, Klein et al 


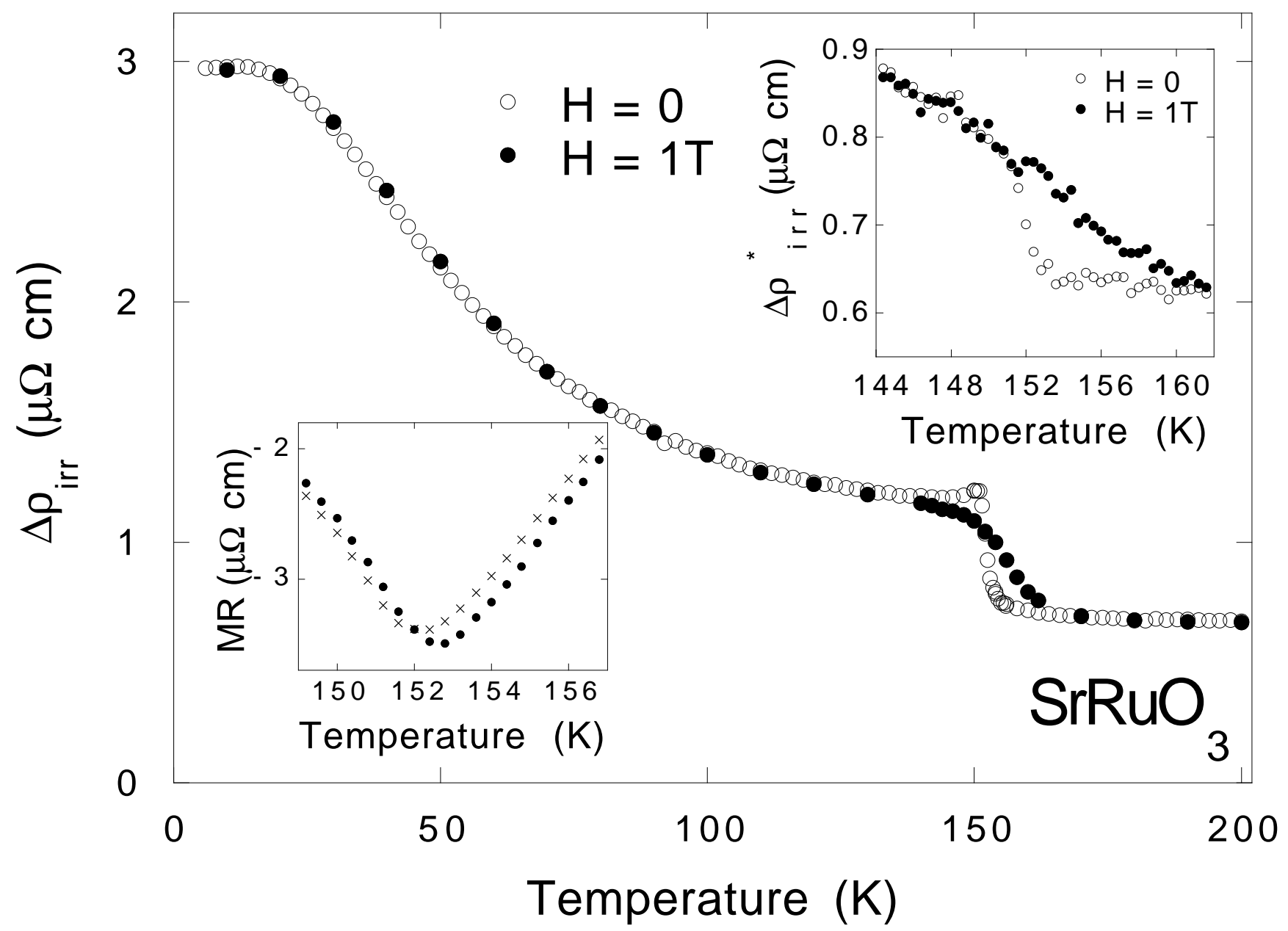

Figure 3, Klein et al 